\title{
Investigation of single valued motions in the vibro-impact system in case of harmonic force
}

\author{
K. Ragulskis ${ }^{1}$, L. Ragulskis ${ }^{2}$ \\ ${ }^{1}$ Kaunas University of Technology, K. Donelaičio str. 73, LT-44249, Kaunas, Lithuania \\ ${ }^{2}$ Department of Systems Analysis, Faculty of Informatics, Vytautas Magnus University, \\ Vileikos str. 8, LT-44404, Kaunas, Lithuania \\ ${ }^{1}$ Corresponding author \\ E-mail: ${ }^{1}$ kazimieras3@hotmail.com,2l.ragulskis@if.vdu.lt \\ Received 30 March 2021; received in revised form 15 June 2021; accepted 25 June 2021 \\ DOI https://doi.org/10.21595/jmeacs.2021.22021 \\ Check for updates \\ Copyright (C) 2021 K. Ragulskis, et al. This is an open access article distributed under the Creative Commons Attribution License, which \\ permits unrestricted use, distribution, and reproduction in any medium, provided the original work is properly cited.
}

\begin{abstract}
It is determined that vibro-impact systems in practical applications generate a number of problems because of the fact that in the regimes in which steady state motions take place multivalued motions are observed. Here it is shown that in a definite investigated case multivalued stable regimes of motion do not exist in the system. Here the investigation of single valued solutions is performed. They are especially useful for practical applications according to the performance of impact processes. The presented graphical relationships enable to choose the regimes suitable for practical applications that are with decaying impacts as well as stationary stable regimes.
\end{abstract}

Keywords: vibro impact system, single valued regimes, region of existence, eigenfrequencies and forced frequencies.

\section{Introduction}

In practical applications in engineering various dynamic regimes of motion of vibro-impact systems take place. An important problem is to investigate those regimes of motion, which are used in engineering applications.

In this paper dynamics in some typical regimes of motion are investigated in detail. Vibrations excited by a force of the system performing vibrations with impacts with excitation by a harmonic force are analysed. Graphical results of investigation for the values of parameters which are typical for the vibrating system were obtained and are shown in detail.

The results of investigation are used in the process of design of systems performing vibrations with impacts and they are applied in different types of technological processes and machines of different types.

Investigation of resonances in vehicle dynamics is presented in [1]. Impacts under periodic and transient excitations are investigated in [2]. Problem of stabilisation of periodic nonlinear systems is solved in [3]. Mechanical systems in which impacts take place are investigated in [4]. Periodic orbits in mechanical systems with impacts are presented in [5]. Investigation of the vibro-impact system and the energy transfer mechanism in it is performed in [6]. Analysis of particle impact with a wall is performed in [7]. Frequency analysis of the system performing vibrations is performed in [8]. Investigation of dynamics of a pendulum mechanism is performed in [9]. Systems with piecewise-linear type of nonlinearity are investigated in [10]. Investigation of resonant zones of a dynamical system is performed in [11]. Analysis of the Sommerfeld effect in a nonlinear oscillator is presented in [12]. Investigation of isolated resonances in a dynamical system is performed in [13]. Analysis of vibro-impact mechanism is presented in [14]. All the references are essential in understanding the current status of investigations of essentially nonlinear mechanical systems.

In this paper first of all model of the system performing vibrations with impacts is presented. The obtained results for several typical regimes of motion of the system are investigated and described in detail. Typical frequency regions with single valued regimes of motion are 
determined.

The presented results of investigation are used for the design of systems performing vibrating motions with impacts as well as mechanisms of various types. In this paper the phenomenon of impact is investigated and it takes place in the process of operation of various types of mechanical systems and mechanisms as well as machines.

\section{Model of the investigated vibro-impact system}

Dynamics of the system performing vibrations with impacts is governed by the differential equation:

$m \ddot{x}+H \dot{x}+C x=F \sin \omega t$, when the displacement $x$ is less than zero,

where $m$ denotes the mass, $H$ denotes the viscous damping coefficient, $C$ denotes the stiffness coefficient, $F$ denotes the excitation amplitude, $\omega$ denotes the frequency, $t$ denotes the time variable, the dot above the quantity indicates derivative by $t$.

Process of impact is described as:

$\dot{x}^{+}=-R \dot{x}^{-}$, when the displacement $x$ is equal to zero,

where by $R$ the restitution coefficient is denoted, by superscript minus the quantity before impact is denoted, by superscript plus the quantity after impact is denoted.

In non dimensional form Eq. (1) becomes:

$x^{\prime \prime}+2 h x^{\prime}+x=f \sin v \tau$.

In non dimensional form Eq. (2) becomes:

$x^{\prime+}=-R x^{\prime-}$.

In the performed investigations the exciting force is denoted as:

$\bar{f}=f \sin v \tau$.

Motions of the system in regimes that are periodic are investigated and the following graphical representations are presented: $\bar{f}$ as function of $\tau, x$ as function of $\tau, x^{\prime}$ as function of $\tau, x^{\prime}$ as function of $x$.

Further vibrations of the vibro impact system for several typical regimes of motions performing steady state vibrations are investigated.

\section{Dynamics in periodic regimes of motion for low frequency of excitation}

Graphical relationships for three typical values of $v$ are shown in the Figs. 1, 2 and 3 in order of increasing values of $v$.

Fig. 1 shows variation of force in time, variation of displacement in time, variation of velocity in time and variation of velocity as function of displacement for $v=1 / 6$. It is seen that a very great number of multiple very fastly decaying impacts take place.

Fig. 2 shows variation of force in time, variation of displacement in time, variation of velocity in time and variation of velocity as function of displacement for $v=1 / 4$. It is seen that a great number of multiple fastly decaying impacts take place.

Fig. 3 shows variation of force in time, variation of displacement in time, variation of velocity in time and variation of velocity as function of displacement for $v=1 / 2$. It is seen that multiple decaying impacts take place. 


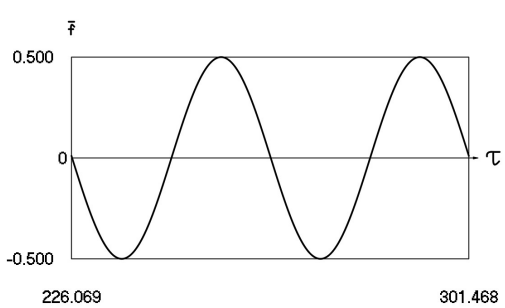

a) Exciting force

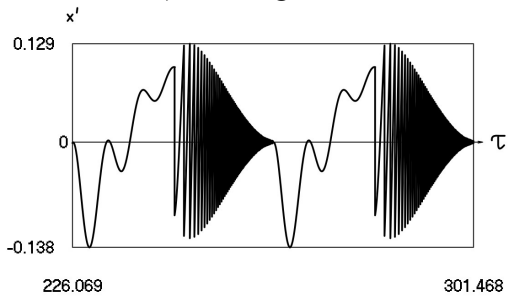

c) Variation of velocity

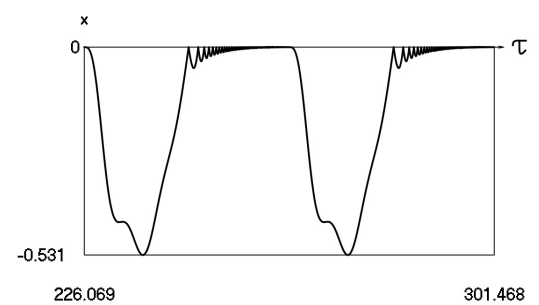

b) Variation of displacement

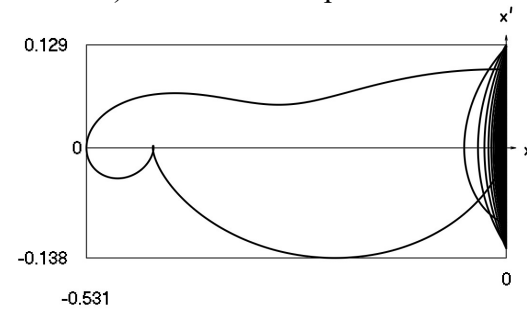

d) Relationship between velocity of the system and its displacement

Fig. 1. Motion in periodic regime at $v=1 / 6, f=-0.5, h=0.1, R=0.975$

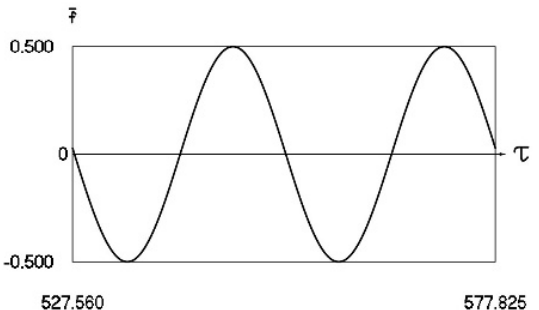

a) Exciting force

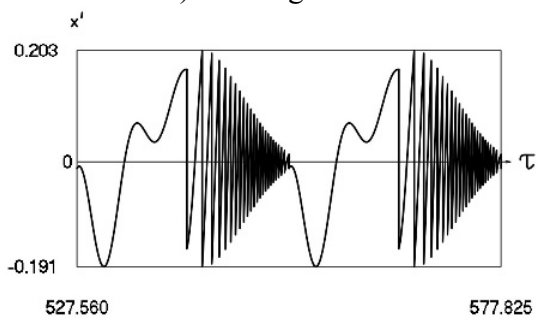

c) Variation of velocity

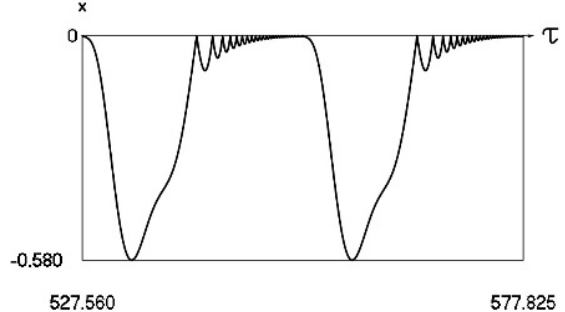

b) Variation of displacement

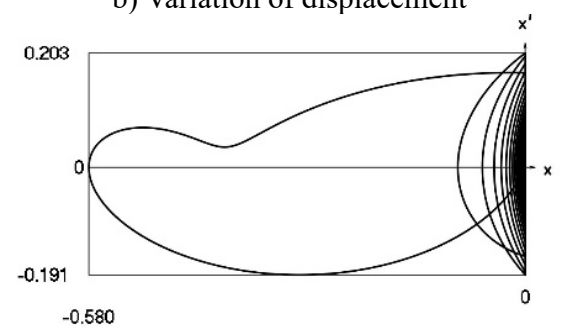

d) Relationship between velocity of the system and its displacement

Fig. 2. Motion in periodic regime at $v=1 / 4, f=-0.5, h=0.1, R=0.94$

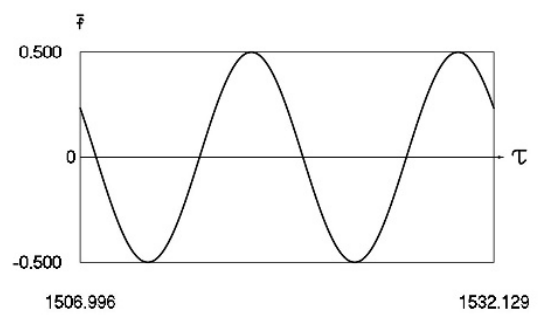

a) Exciting force

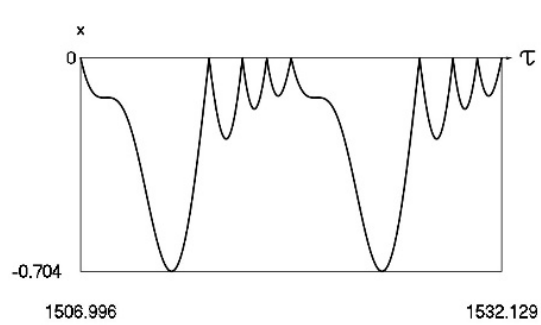

b) Variation of displacement 


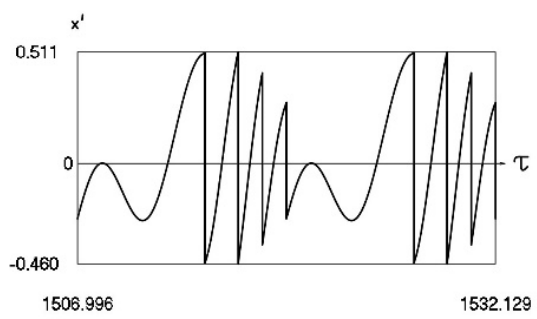

c) Variation of velocity

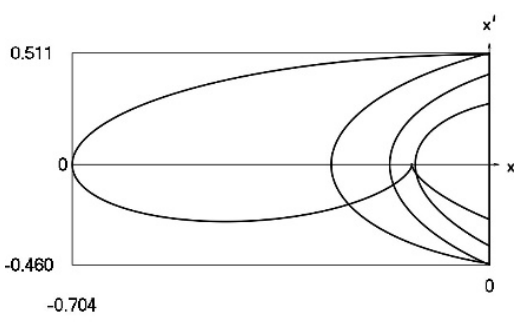

d) Relationship between velocity of the system and its displacement

Fig. 3. Motion in periodic regime at $v=1 / 2, f=-0.5, h=0.1, R=0.9$

It is observed that during the motion in a period of force a number of impacts are observed. With the value of $v$ becoming smaller in general the number of impacts becomes higher. Decaying impacts are clearly observed in the obtained results. Thus it can be considered that cyclic motion of the vibro-impact system takes place.

\section{Dynamics in periodic regimes of motion for frequency around four}

Graphical relationships for five typical values of $v$ are shown in the Figs. 4-8. The first Figure is near to the region of single valued motions from the external side of this region, while the second Figure is near to the region of single valued motions from the internal side and thus they approximately represent the lower boundary of the region of single valued motions. The third Figure represents motion at the approximately optimal frequency of excitation. The fourth Figure is near to the region of single valued motions from the internal side of this region, while the fifth Figure is near to the region of single valued motions from the external side and thus they approximately represent the upper boundary of the region of single valued motions.

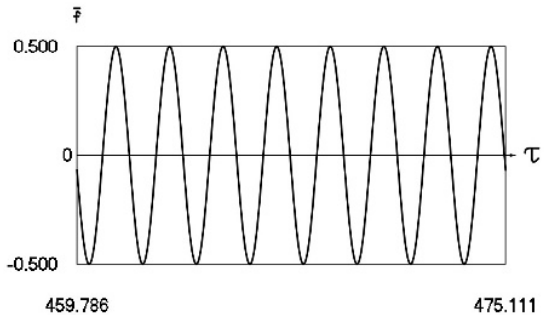

a) Exciting force

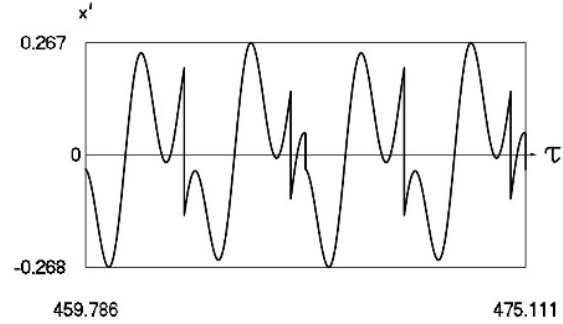

c) Variation of velocity

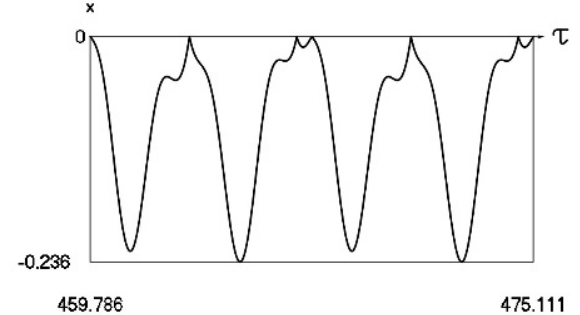

b) Variation of displacement

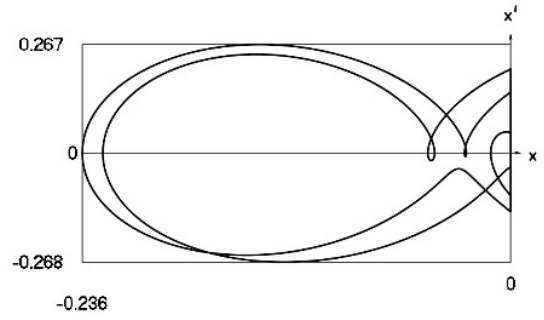

d) Relationship between velocity of the system and its displacement

Fig. 4. Motion in periodic regime at $v=3.28, f=-0.5, h=0.1, R=0.7$

Fig. 4 shows variation of force in time, variation of displacement in time, variation of velocity in time and variation of velocity as function of displacement for $v=3.28$. It is seen that the system is operating at the lower border outside of the recommended zone of single valued motions.

Fig. 5 shows variation of force in time, variation of displacement in time, variation of velocity 
in time and variation of velocity as function of displacement for $v=3.3$. It is seen that the system is operating at the lower border inside of the recommended zone of single valued motions.

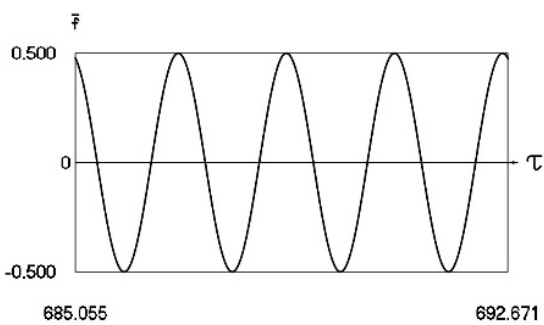

a) Exciting force

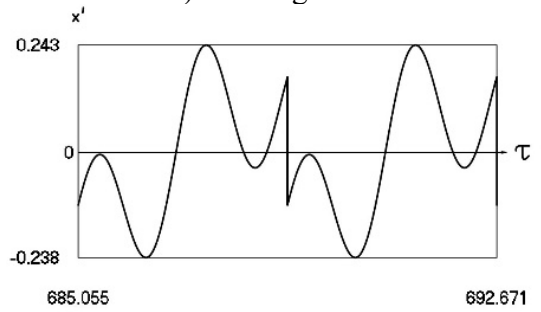

c) Variation of velocity

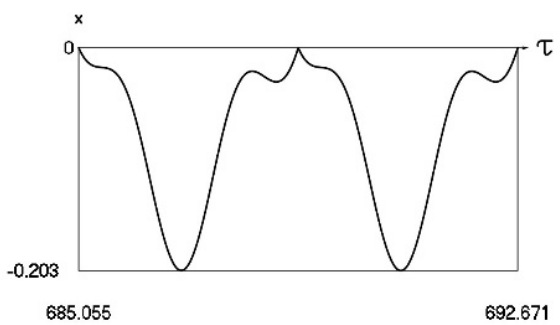

b) Variation of displacement

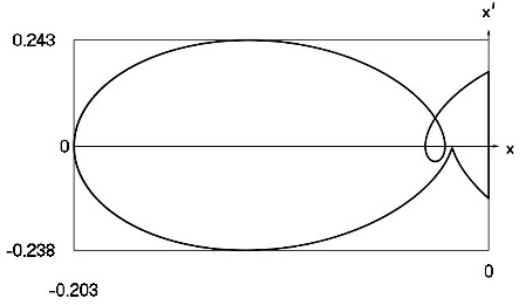

d) Relationship between velocity of the system and its displacement

Fig. 5. Motion in periodic regime at $v=3.3, f=-0.5, h=0.1, R=0.7$

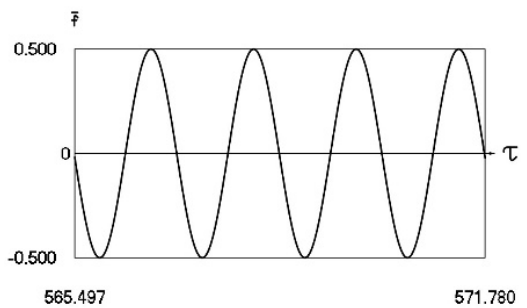

a) Exciting force

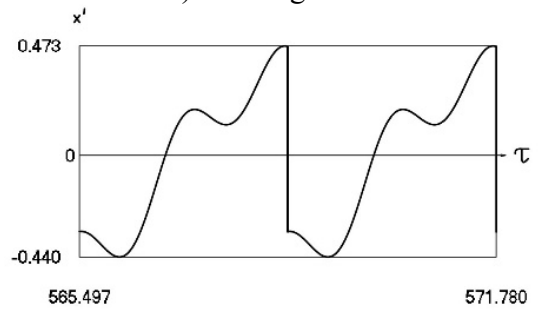

c) Variation of velocity

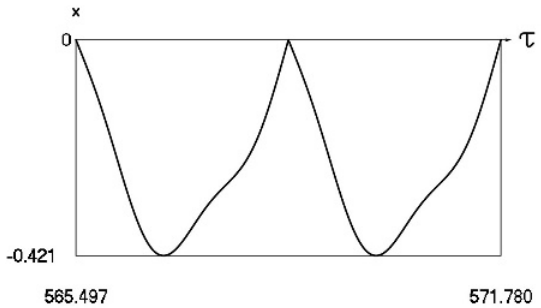

b) Variation of displacement

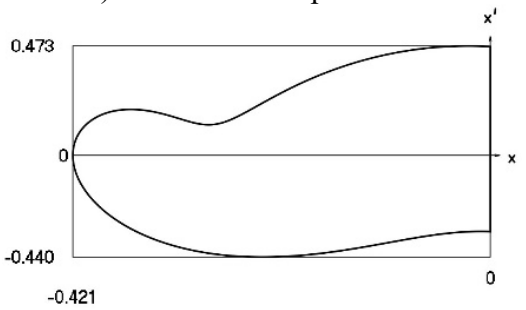

d) Relationship between velocity of the system and its displacement

Fig. 6. Motion in periodic regime at $v=4,6, f=-0.5, h=0.1, R=0.7$

Fig. 6 shows variation of force in time, variation of displacement in time, variation of velocity in time and variation of velocity as function of displacement for $v=4$. It is seen that the system is operating at recommended frequency.

Fig. 7 shows variation of force in time, variation of displacement in time, variation of velocity in time and variation of velocity as function of displacement for $v=4.6$. It is seen that the system is operating at the upper border inside of the recommended zone of single valued motions.

Fig. 8 shows variation of force in time, variation of displacement in time, variation of velocity in time and variation of velocity as function of displacement for $v=4.7$. It is seen that the system 
is operating at the upper border outside of the recommended zone of single valued motions.

From the obtained graphical relationships the recommended zone of single valued motions can be seen.

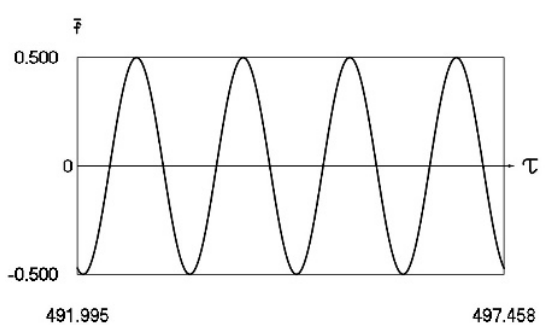

a) Exciting force

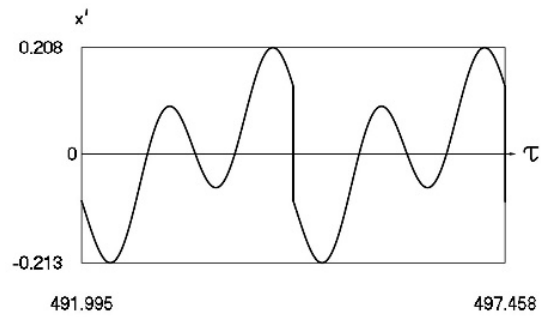

c) Variation of velocity

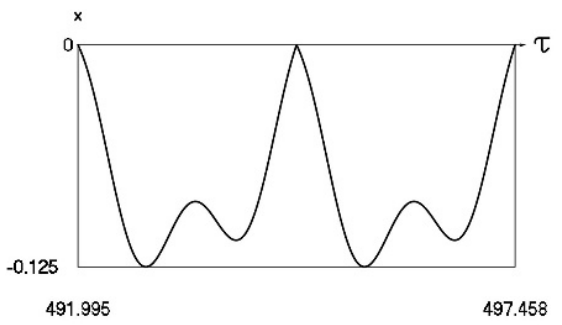

b) Variation of displacement

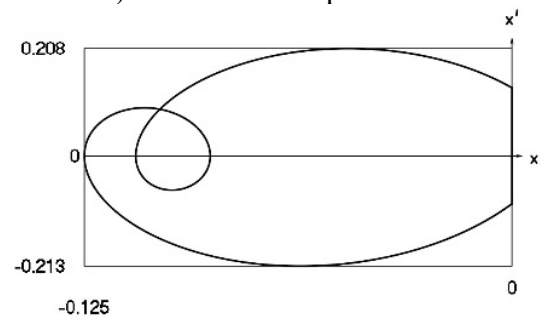

d) Relationship between velocity of the system and its displacement

Fig. 7. Motion in periodic regime at $v=4.26, f=-0.5, h=0.1, R=0.7$

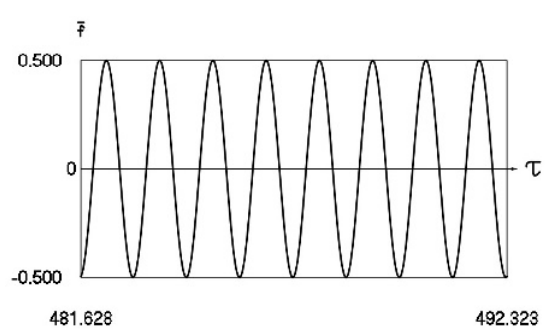

a) Exciting force

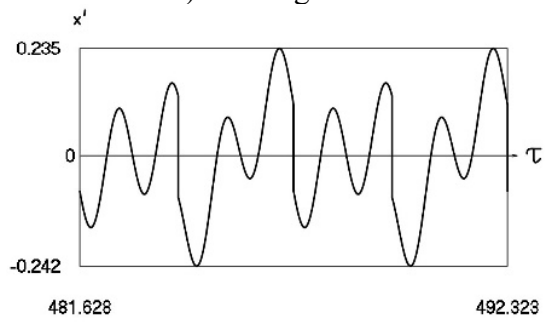

c) Variation of velocity

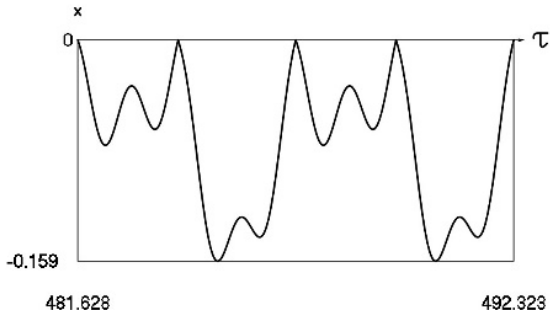

b) Variation of displacement

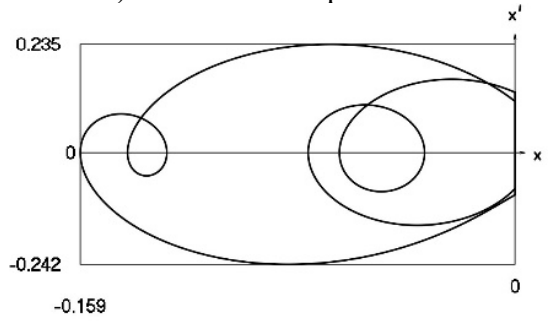

d) Relationship between velocity of the system and its displacement

Fig. 8. Motion in periodic regime at $v=4.7, f=-0.5, h=0.1, R=0.7$

\section{Dynamics in periodic regimes of motion for frequency around six}

Graphical relationships for five typical values of $v$ are shown in the Figs. 9-13. The first Figure is near to the region of single valued motions from the external side of this region, while the second Figure is near to the region of single valued motions from the internal side and thus they approximately represent the lower boundary of the region of single valued motions. The third 
Figure represents motion at the approximately optimal frequency of excitation. The fourth Figure is near to the region of single valued motions from the internal side of this region, while the fifth Figure is near to the region of single valued motions from the external side and thus they approximately represent the upper boundary of the region of single valued motions.

Fig. 9 shows variation of force in time, variation of displacement in time, variation of velocity in time and variation of velocity as function of displacement for $v=5.28$. It is seen that the system is operating at the lower border outside of the recommended zone of single valued motions.

Fig. 10 shows variation of force in time, variation of displacement in time, variation of velocity in time and variation of velocity as function of displacement for $v=5.3$. It is seen that the system is operating at the lower border inside of the recommended zone of single valued motions.

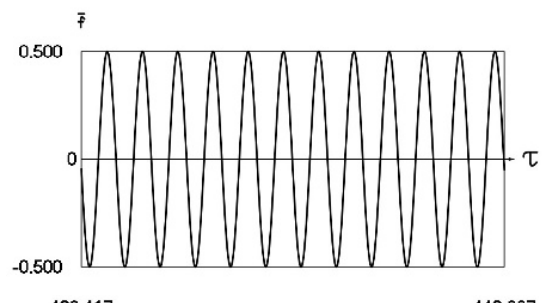

a) Exciting force

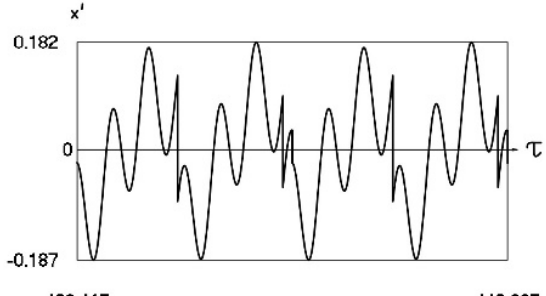

c) Variation of velocity

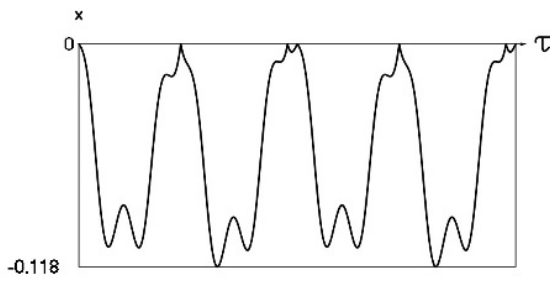

428.417

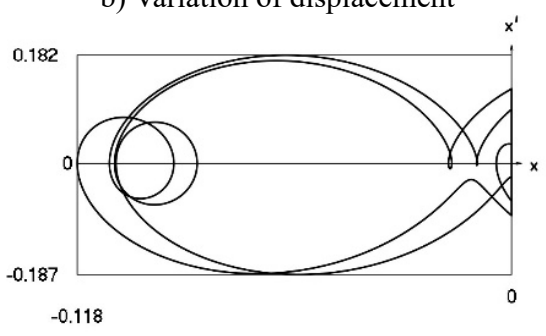

d) Relationship between velocity of the system and its displacement

Fig. 9. Motion in periodic regime at $v=5.28, f=-0.5, h=0.1, R=0.7$

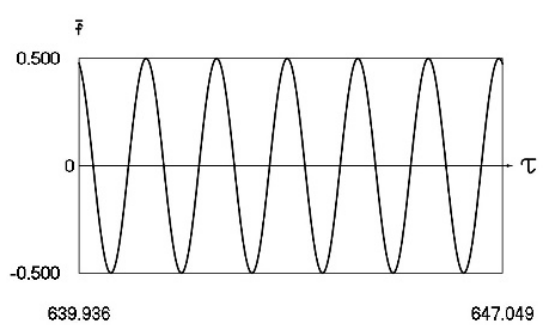

a) Exciting force

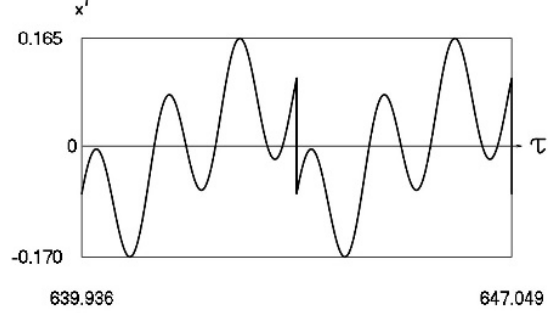

c) Variation of velocity

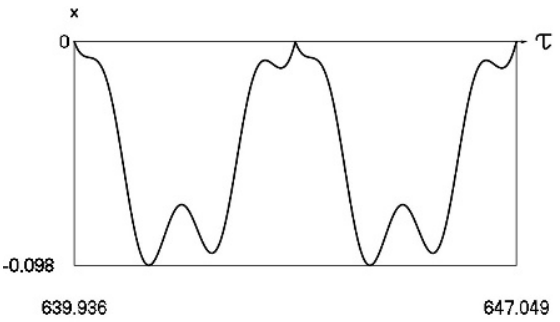

b) Variation of displacement

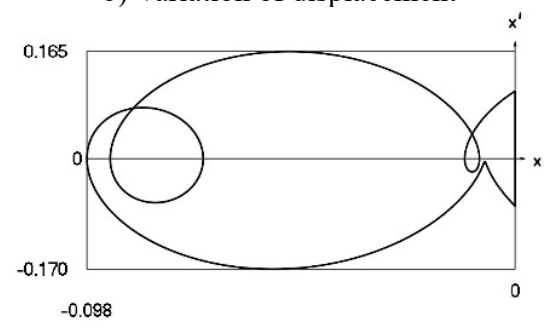

d) Relationship between velocity of the system and its displacement

Fig. 10. Motion in periodic regime at $v=5.3, f=-0.5, h=0.1, R=0.7$ 
Fig. 11 shows variation of force in time, variation of displacement in time, variation of velocity in time and variation of velocity as function of displacement for $v=6$. It is seen that the system is operating at recommended frequency.

Fig. 12 shows variation of force in time, variation of displacement in time, variation of velocity in time and variation of velocity as function of displacement for $v=6.6$. It is seen that the system is operating at the upper border inside of the recommended zone of single valued motions.

Fig. 13 shows variation of force in time, variation of displacement in time, variation of velocity in time and variation of velocity as function of displacement for $v=6.7$. It is seen that the system is operating at the upper border outside of the recommended zone of single valued motions.

From the obtained graphical relationships the recommended zone of single valued motions can be seen.

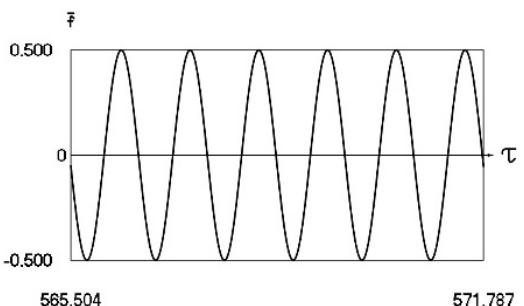

a) Exciting force

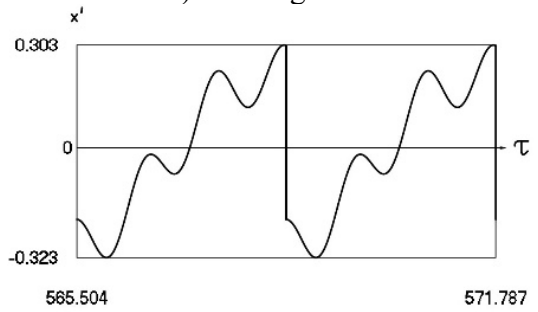

c) Variation of velocity

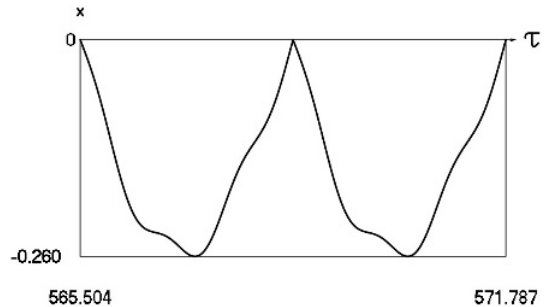

b) Variation of displacement

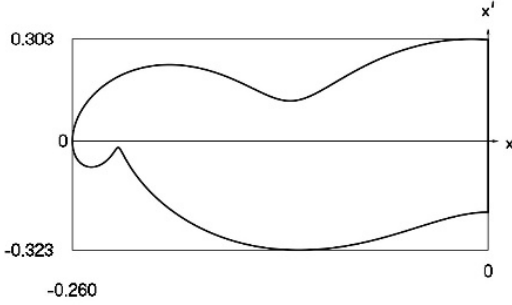

d) Relationship between velocity of the system and its displacement

Fig. 11. Motion in periodic regime at $v=6, f=-0.5, h=0.1, R=0.7$

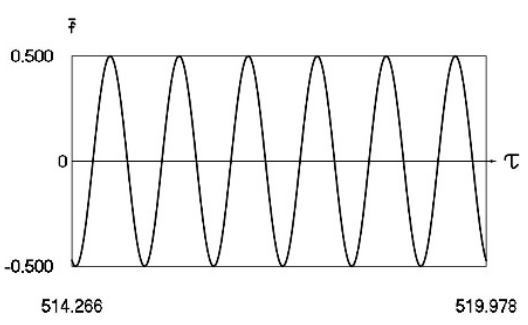

a) Exciting force

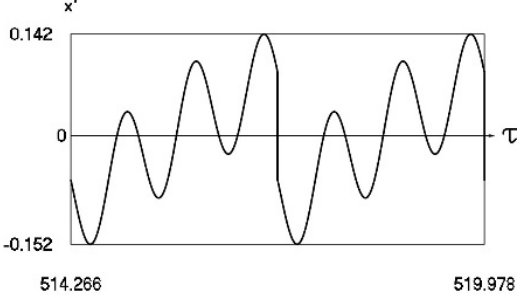

c) Variation of velocity

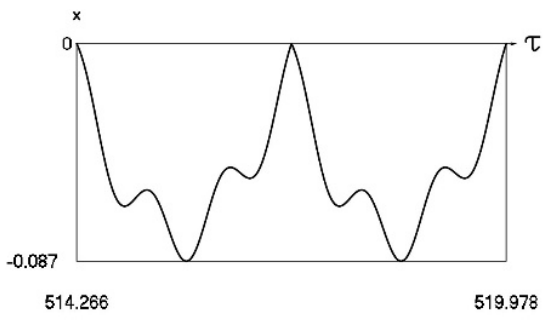

b) Variation of displacement

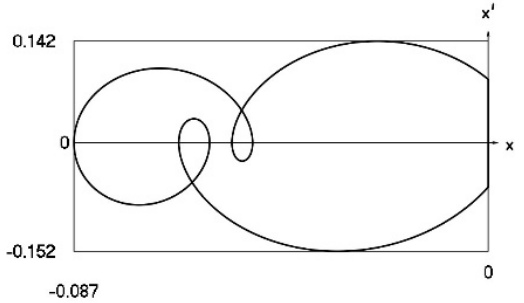

d) Relationship between velocity of the system and its displacement

Fig. 12. Motion in periodic regime at $v=6.6, f=-0.5, h=0.1, R=0.7$ 


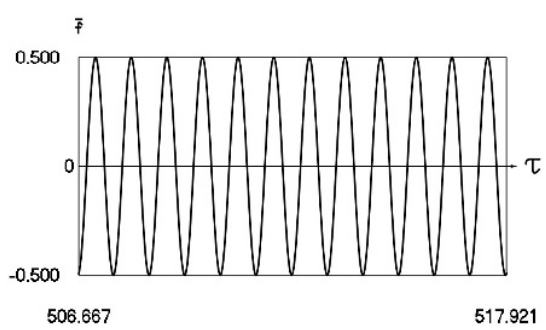

a) Exciting force

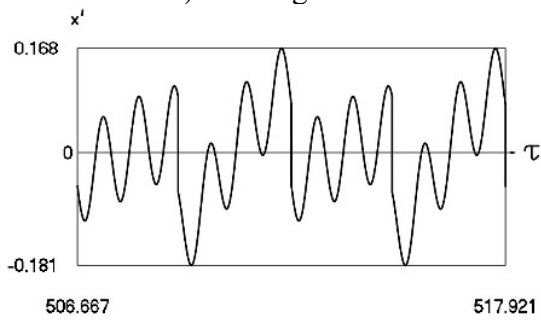

c) Variation of velocity

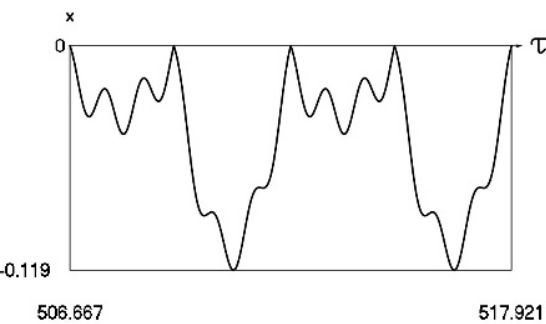

b) Variation of displacement

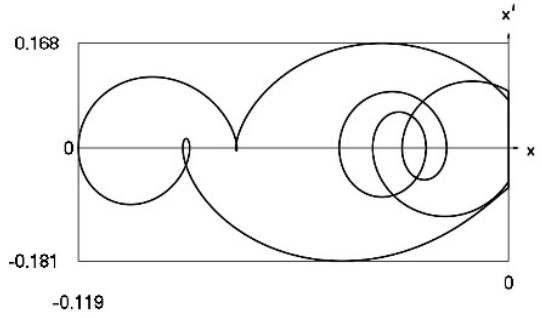

d) Relationship between velocity of the system and its displacement

Fig. 13. Motion in periodic regime at $v=6.7, f=-0.5, h=0.1, R=0.7$

\section{Regions of optimal behavior of the system in periodic regimes of motion}

Minimum and maximum values of basic parameters determining dynamic behavior of the investigated vibro-impact system are investigated as functions of frequency of excitation. Frequency regions were minimum and maximum values coincide correspond to regions of single valued motions, while to the left and right of those regions multi valued motions take place. Single valued motions are important in practical applications and thus this paper is devoted to their investigation.

Parameters describing the dynamics at $v=2$ are shown in Fig. 14.

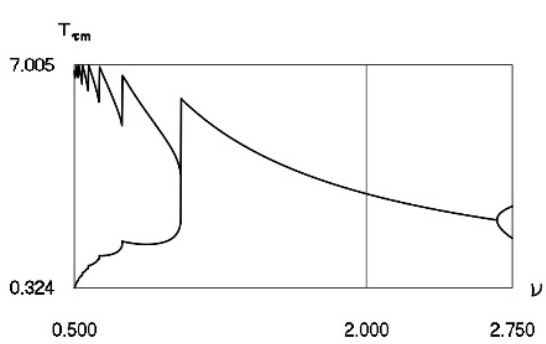

a) Intervals between impacts

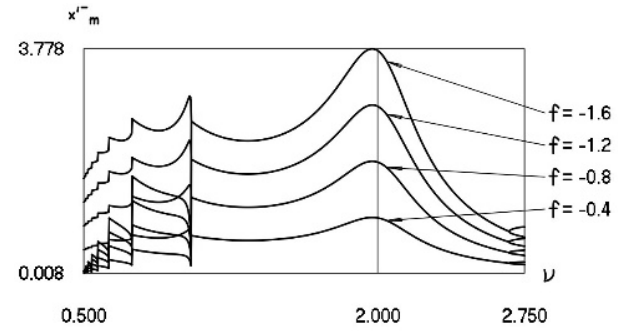

b) Derivatives of displacement before the moment of impact

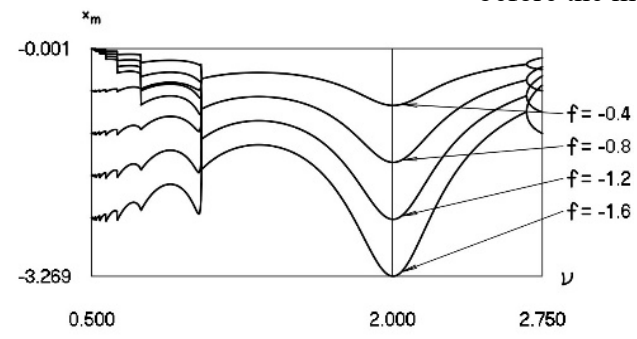

c) Minimum values of displacements in the intervals between impacts

Fig. 14. Minimum values and maximum values for $h=0.1, R=0.7$ in the vicinity of $v=2$ 
In Fig. 14 the values of intervals between impacts, velocities before impact and minimum values of displacements in the intervals between impacts are represented.

Parameters describing the dynamics at $v=4$ are shown in Fig. 15.

In Fig. 15 the values of intervals between impacts, velocities before impact and minimum values of displacements in the intervals between impacts are represented.

Parameters describing the dynamics at $v=6$ are shown in Fig. 16.

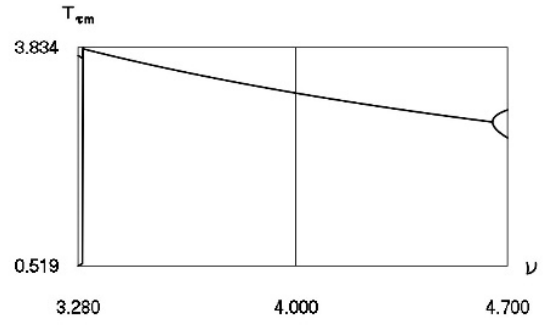

a) Intervals between impacts

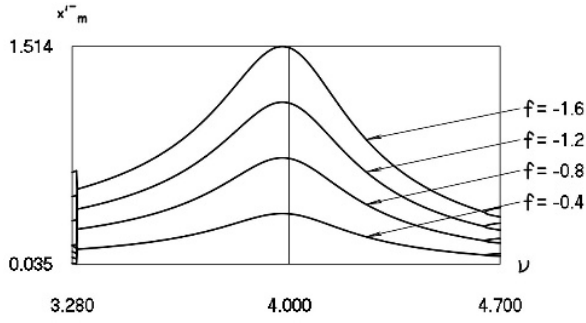

b) Derivatives of displacement before the moment of impact

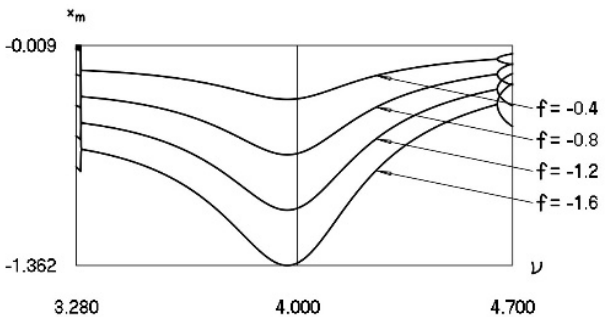

c) Minimum values of displacements in the intervals between impacts

Fig. 15. Minimum values and maximum values for $h=0.1, R=0.7$ in the vicinity of $v=4$

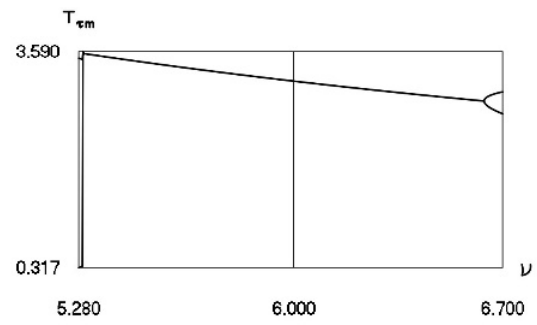

a) Intervals between impacts

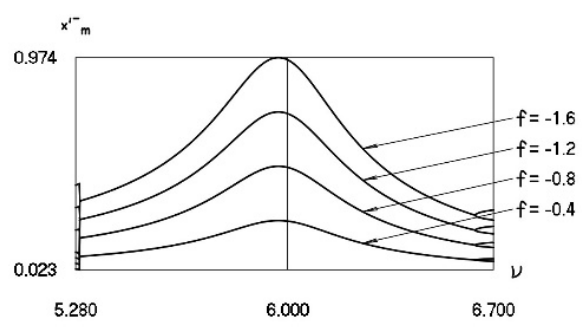

b) Derivatives of displacement before the moment of impact

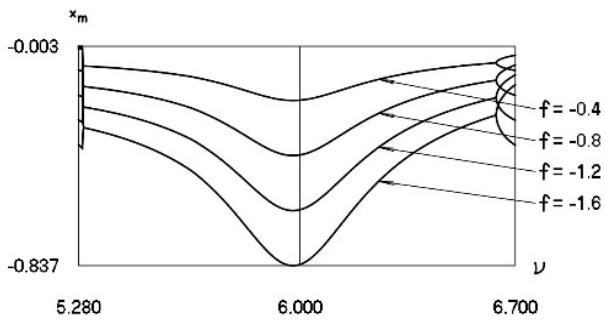

c) Minimum values of displacements in the intervals between impacts

Fig. 16. Minimum values and maximum values for $h=0.1, R=0.7$ in the vicinity of $v=6$

In Fig. 16 the values of intervals between impacts, velocities before impact and minimum values of displacements in the intervals between impacts are represented.

Results at $f=-0.4, f=-0.8, f=-1.2, f=-1.6$ are presented in the previous three figures. 
Intervals between impacts are mutually similar for the four values of $f$. Recommended frequency regions are clearly seen in the presented figures.

\section{Conclusions}

Vibro impact systems in practical applications generate a number of problems because of the fact that in the regimes in which steady state motions take place multivalued motions are observed. In the paper it is determined that this can be avoided by choosing some of the parameters of the system. This is very important in practical applications creating vibro-impact systems. Thus, it is shown that in a definite investigated case multivalued stable regimes of motion do not exist in the system. The investigation of single valued solutions is performed.

System performing vibrating motions with impacts excited by a harmonic force is analysed, especially those regimes of motion, which are important in engineering applications. Dynamics in some typical regimes of motion is investigated in detail.

Regimes of motion for frequencies of excitation $v=1 / 6 ; 1 / 4 ; 1 / 2$ are investigated. Those regimes are with multiple decaying impacts. Thus, it is concluded that for low frequencies of excitation impacts have the character of decaying type.

The size according to the frequency of excitation of separate optimal regions of operation about $v=2 ; 4 ; 6$ in which there exist single valued solutions is determined. Minimum and maximum values of basic parameters determining dynamic behavior of the investigated vibro impact system are investigated as functions of frequency of excitation. Frequency regions were minimum and maximum values coincide correspond to regions of single valued motions, while to the left and right of those regions multi valued motions take place. Single valued motions are important in practical applications and thus this paper is devoted to their investigation.

The results of investigation are applied in the process of design of systems performing vibrating motions with impacts and are used in their applications in different technological processes and machines of different types.

\section{References}

[1] W. V. Wedig, "New resonances and velocity jumps in nonlinear road-vehicle dynamics," Procedia IUTAM, Vol. 19, pp. 209-218, 2016, https://doi.org/10.1016/j.piutam.2016.03.027

[2] T. Li, E. Gourc, S. Seguy, and A. Berlioz, "Dynamics of two vibro-impact nonlinear energy sinks in parallel under periodic and transient excitations," International Journal of Non-Linear Mechanics, Vol. 90, pp. 100-110, Apr. 2017, https://doi.org/10.1016/j.ijnonlinmec.2017.01.010

[3] V. A. Zaitsev, "Global asymptotic stabilization of periodic nonlinear systems with stable free dynamics," Systems and Control Letters, Vol. 91, pp. 7-13, May 2016, https://doi.org/10.1016/j.sysconle.2016.01.004

[4] H. Dankowicz and E. Fotsch, "On the analysis of chatter in mechanical systems with impacts," Procedia IUTAM, Vol. 20, pp. 18-25, 2017, https://doi.org/10.1016/j.piutam.2017.03.004

[5] S. Spedicato and G. Notarstefano, "An optimal control approach to the design of periodic orbits for mechanical systems with impacts," Nonlinear Analysis: Hybrid Systems, Vol. 23, pp. 111-121, Feb. 2017, https://doi.org/10.1016/j.nahs.2016.08.009

[6] W. Li, N. E. Wierschem, X. Li, and T. Yang, "On the energy transfer mechanism of the single-sided vibro-impact nonlinear energy sink," Journal of Sound and Vibration, Vol. 437, pp. 166-179, Dec. 2018, https://doi.org/10.1016/j.jsv.2018.08.057

[7] J. S. Marshall, "Modeling and sensitivity analysis of particle impact with a wall with integrated damping mechanisms," Powder Technology, Vol. 339, pp. 17-24, Nov. 2018, https://doi.org/10.1016/j.powtec.2018.07.097

[8] E. Salahshoor, S. Ebrahimi, and Y. Zhang, "Frequency analysis of a typical planar flexible multibody system with joint clearances," Mechanism and Machine Theory, Vol. 126, pp. 429-456, Aug. 2018, https://doi.org/10.1016/j.mechmachtheory.2018.04.027

[9] U. Starossek, "Forced response of low-frequency pendulum mechanism," Mechanism and Machine Theory, Vol. 99, pp. 207-216, May 2016, https://doi.org/10.1016/j.mechmachtheory.2016.01.004 
[10] S. Wang, L. Hua, C. Yang, Y. O. Zhang, and X. Tan, "Nonlinear vibrations of a piecewise-linear quarter-car truck model by incremental harmonic balance method," Nonlinear Dynamics, Vol. 92, No. 4, pp. 1719-1732, Jun. 2018, https://doi.org/10.1007/s11071-018-4157-6

[11] P. Alevras, S. Theodossiades, and H. Rahnejat, "On the dynamics of a nonlinear energy harvester with multiple resonant zones," Nonlinear Dynamics, Vol. 92, No. 3, pp. 1271-1286, May 2018, https://doi.org/10.1007/s11071-018-4124-2

[12] A. Sinha, S. K. Bharti, A. K. Samantaray, G. Chakraborty, and R. Bhattacharyya, "Sommerfeld effect in an oscillator with a reciprocating mass," Nonlinear Dynamics, Vol. 93, No. 3, pp. 1719-1739, Aug. 2018, https://doi.org/10.1007/s11071-018-4287-x

[13] G. Habib, G. I. Cirillo, and G. Kerschen, "Isolated resonances and nonlinear damping," Nonlinear Dynamics, Vol. 93, No. 3, pp. 979-994, Aug. 2018, https://doi.org/10.1007/s11071-018-4240-z

[14] K. Ragulskis and L. Ragulskis, "Vibroimpact mechanism in one separate case," Mathematical Models in Engineering, Vol. 5, No. 2, pp. 56-63, Jun. 2019, https://doi.org/10.21595/mme.2019.20818

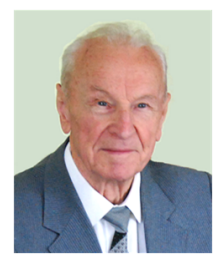

Kazimieras Ragulskis is a member of the Academy of Sciences of the USSR (later of the Russian Academy of Sciences) and of the Lithuanian Academy of Sciences. His research field is precise vibromechanics and vibroengineering. That is vibrations and waves in nonlinear dynamical systems.

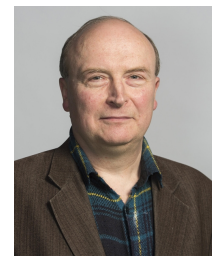

Liutauras Ragulskis is from Vytautas Magnus University, where he is a research associate. His research is related with numerical investigations of systems performing vibrations. 